\title{
System identification of smart structures under high impact loads
}

\author{
Y. Kim, T. El-Korchi \& K. S. Arsava \\ Department of Civil and Environmental Engineering, \\ Worcester Polytechnic Institute (WPI), Worcester, MA, USA
}

\begin{abstract}
This paper proposes a fuzzy model for predicting nonlinear behaviour of smart structures. The parameters of the fuzzy model are trained using the backpropagation neural network and least squares algorithms. To demonstrate the effectiveness of the proposed Takagi-Sugeno (TS) fuzzy model, a structure equipped with magnetorheological (MR) dampers is constructed and investigated. Various levels of high impact loads and current signals are used as disturbances and control signals, respectively. It is demonstrated from the experimental studies that the proposed TS fuzzy model is effective in estimating the high impact responses of the smart structural systems subjected to a variety of high impact loads.

Keywords: Takagi-Sugeno (TS) fuzzy model, neural network, high impact loads, magnetorheological (MR) damper, structural control.
\end{abstract}

\section{Introduction}

In recent years, the application of smart control technology to large-scale civil structures has been proposed because it combines the best features of both the passive and active control systems. In particular, magnetorheological (MR) damper has attracted a great deal of attention to structural engineering due to many advantages such as reliability, cost-effectiveness, etc. [1]. Although the vast majority of the studies for MR dampers have led to a great understanding of controllable fluid damper at low velocities and frequencies [2], behavior of MR dampers under high impact loads are relatively unknown [3-6]. The main focus of their studies is on behavior of MR damper itself under impact loads. However, there is no available approach in the literature about how to model an integrated 
structure-MR damper system under high impact loads (including the interaction effects between a structure and an MR damper). To apply MR dampers to structures under high impact loads, fundamental understanding of an integrated structure-MR damper systems as well as MR damper itself needs to be addressed. In addition, an effective control algorithm should be designed in order to effectively operate the MR damper within structural systems under high impact forces [7]. To develop the smart control algorithms, it is desired to develop a mathematical model for describing nonlinear behavior of the integrated structure equipped with MR dampers $[8,9]$. Therefore, the objective of this study is to develop mathematical models for structural systems equipped with MR dampers under high impact loads.

\section{System identification}

\subsection{Takagi-Sugeno (TS) fuzzy model}

In 1985, Takagi and Sugeno [10] developed a systematic methodology for a fuzzy reasoning using linear functions in the consequent part. Because the TSfuzzy model uses linear functions in the consequent part, the defuzzification procedure is not required. A typical fuzzy rule for the TS fuzzy model has the form

$$
\begin{gathered}
\mathrm{R}_{j} \text { : If } z_{\mathrm{FZ}}^{1} \text { is } p_{1, j} \text { and } z_{\mathrm{FZ}}^{2} \text { is } p_{2, j} \text { and } \cdots \text { and } z_{\mathrm{FZ}}^{i} \text { is } p_{i, j} \\
\text { Then, } \mathbf{y}_{j}(k)=\sum_{i=1}^{n} \mathbf{a}_{i, j} \mathbf{y}_{j}(k-i)+\sum_{i=1}^{m} \mathbf{b}_{i, j} \mathbf{u}_{j}(k-i),
\end{gathered}
$$

where $\mathrm{R}_{j}$ is the $j^{\text {th }}$ rule of the fuzzy model; $z_{\mathrm{FZ}}^{i}$ is the premise variable; $\mathrm{u}(\mathrm{k})$ and $\mathrm{y}(\mathrm{k})$ are the input and output, respectively; $\mathbf{a}_{i, j}$ and $\mathbf{b}_{i, j}$ are the consequent parameters to be estimated; $n$ and $m$ are the number of delay steps in the output and input signals, respectively. The TS fuzzy model-based reasoning is to simply compute weighted mean values

$$
\mathbf{y}_{\text {final }}=\frac{\sum_{j=1}^{N_{r}} \mathbf{w}_{j} \mathbf{y}_{j}(k)}{\sum_{j=1}^{N_{r}} \mathbf{w}_{j}},
$$

where $N r$ is the number of the fuzzy rules and

$$
\mathbf{w}_{j}=\prod_{i=1}^{n} \mu_{i, j}\left(z_{\mathrm{FZ}}^{i}\right) \text {. }
$$

where $\mu_{i, j}\left(z_{\mathrm{FZ}}^{i}\right)$ is the grades of membership of $z_{\mathrm{FZ}}^{i}$. In this paper, the premise parameters are determined using the fuzzy C-means clustering algorithm [11] while the consequent parameters are optimized via the backpropagation neural network algorithm. 


\subsection{Identification of high impact response}

Figure 1 shows the configuration of the structural system equipped with nonlinear hysteretic actuators. In this study, two MR dampers are implemented into the structural system for impact force attenuations.

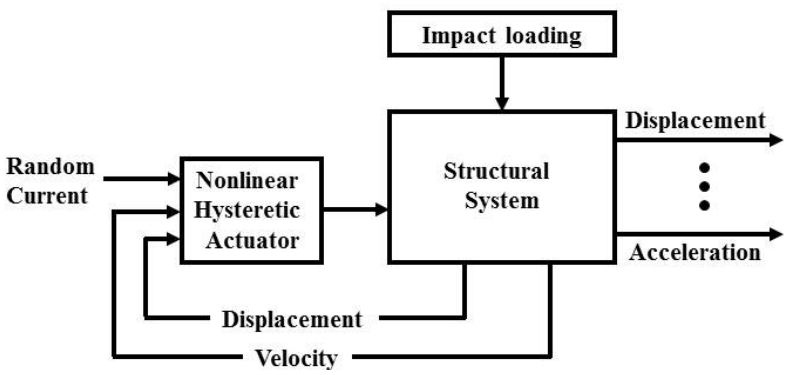

Figure 1: Nonlinear system identification process of integrated structurecontrol system under high impact loads.

A two-input and single output TS fuzzy model is developed such that the fuzzy model effectively predicts the impact responses of the smart structures. The high impact force and current signals are used as the $1^{\text {st }}$ and $2^{\text {nd }}$ input signals while either displacement or acceleration responses are used as the output signal.

\section{Experimental study}

\subsection{Experimental setup}

To demonstrate the effectiveness of the TS fuzzy modelling framework, an experimental setup is established in order to generate many sets of input and output data as shown in Figure 2.
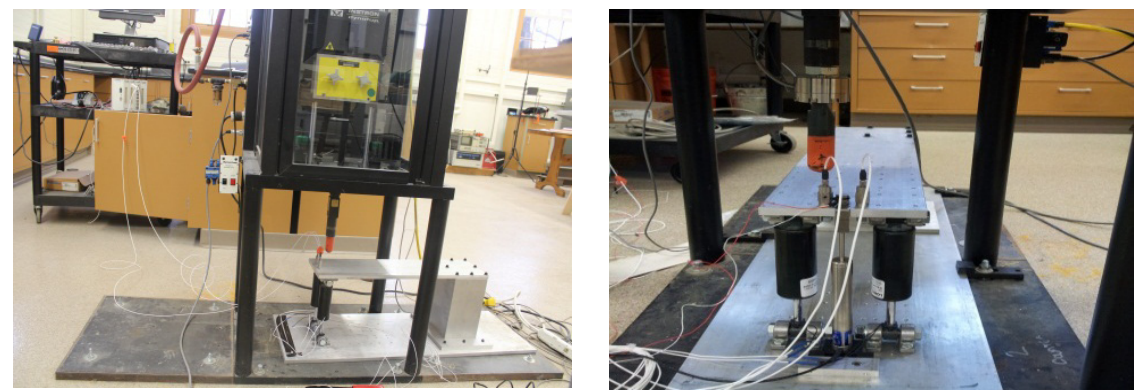

Figure 2: Configuration of an experimental setup to study dynamic behavior of structures equipped with MR dampers. 


\subsection{Results}

Figure 3 compares the measured data with the predicted model. It is clearly seen that the proposed TS fuzzy model effectively predicts nonlinear behavior of the structural system equipped with MR dampers. This demonstrates the feasibility of the TS fuzzy model for structural health monitoring and structural control system design.

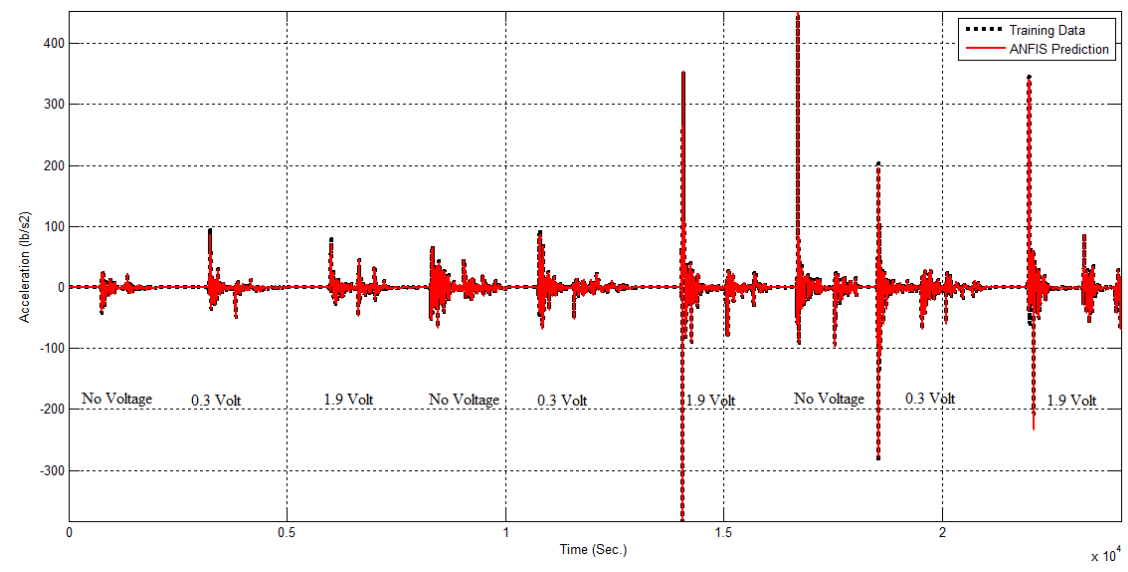

Figure 3: Comparison of the original data with the TS fuzzy model under the high impact loads.

\section{Conclusions}

In this paper, a Takagi-Sugeno (TS) fuzzy model was developed to estimate the nonlinear responses of smart structures under high impact loads. The TS fuzzy model was trained using the backpropagation neural network and least squares algorithms. To demonstrate the effectiveness of the modelling framework, a cantilever beam equipped with two magnetorheological (MR) dampers is manufactured and investigated. The impact forces and currents are used as input signals while displacement and acceleration responses are collected. It is shown from the comparison of the estimated and measured data that the TS fuzzy model is effective in predicting the high impact responses of smart structures.

\section{Reference}

[1] Y. Kim, R. Langari and S. Hurlebaus (2009) Semiactive Nonlinear Control of a Building with a Magnetorheological Damper System, Mechanical Systems and Signal Processing, 23, 300-315.

[2] B.F. Jr. Spencer, S.J. Dyke, M.K. Sain and J.D. Carlson (1997) Phenomenological Model for Magnetorheological Dampers, ASCE Journal of Engineering Mechanics, 123, 230-238. 
[3] M. Ahmadian and J.A. Norris (2008), Experimental Analysis of Magnetorheological Dampers when Subjected to Impact and Shock Loading, Communications in Nonlinear Science and Numerical Simulation, 13, 1987-1985.

[4] H. Hongshen, Q. Suxiang, J. Wang and Z. Li (2009), Performance Simulation and Experimental Evaluation for a magnet-Rheological Damper under Impact Load, Proceedings of the 2008 IEEE International Conference on Robotics and Biomimetics, Bangkok, Thailand, Feb 21-26.

[5] M. Mao, W. Hu, Y.T. Choi and N.M. Wereley (2007), A Magnetorheological Damper with Bifold Valves for Shock and Vibration Mitigation, Journal of Intelligent Material Systems and Structures, 18, $1227-1232$.

[6] J. Wang and Y. Li (2006), Dynamic Simulation and Test Verification of MR Shock Absorber under Impact Load, Journal of Intelligent Material Systems and Structures, 17, 309-314.

[7] R. Mitchell, Y. Kim and T. El-Korchi (2012a) Wavelet-based Neuro-Fuzzy Controller for Vibration Mitigation of High-rise Buildings, Journal of Vibration and Control, in press.

[8] Y. Kim, R. Langari and S. Hurlebaus (2011) MIMO Fuzzy Identification of Building-MR damper System, International Journal of Intelligent and Fuzzy Systems, 22, 1-21.

[9] R. Mitchell, Y. Kim and T. El-Korchi (2012b) System Identification of Smart Structures using Wavelet Neuro-Fuzzy Model, Smart Materials and Structures, in press.

[10] T. Takagi and M. Sugeno (1985), Fuzzy Identification of Systems and Its Applications to Modeling and Control, IEEE Transactions on Systems, Man, and Cybernetics, 15, 116-132.

[11] J.S. Kim, Y. Kim and T. El-Korchi (2012), Fuzzy Identification of Seismically Excited Smart Systems," Structural Seismic Design Optimization and Earthquake Engineering: Formulations and Applications, IGI-Global, Inc., Nikos Lagaros (ed.), 323-341. 